\title{
PERSPECTIVAS
}

Artigo convidado

http://dx.doi.org/10.1590/So034-759020210206

\section{PODE UMA LEI PROGRESSISTA ACELERAR UMA TRAJETORIA DE GRANDE LENTIDÃO? MULHERES NOS CONSELHOS DE ADMINISTRAÇÃO EM PORTUGAL}

Este artigo analisa o impacto do novo marco regulatório que entrou em vigor em Portugal em janeiro de 2018, a chamada lei das cotas de gênero, que determina uma composição mais equilibrada de mulheres e homens nos conselhos de administração das empresas cotadas na Bolsa de Valores Euronext Lisbon e das empresas do setor público. $\mathrm{O}$ artigo começa por contextualizar o debate sobre a sub-representação de mulheres nos conselhos de administração e sobre o novo marco de políticas com efeito vinculativo, introduzido em vários países da União Europeia (UE) e em Portugal. Examina, em seguida, os dados relativos à representação de homens e mulheres nos conselhos de administração das empresas cotadas em bolsa. Conclui-se que a introdução desta medida legislativa de natureza vinculativa em Portugal acelerou o movimento em direção a uma maior representação das mulheres nos conselhos de administração das empresas cotadas em bolsa, representando um novo impulso ao progresso neste domínio, porém lento, gerado pelos anteriores incentivos que visaram estimular a ação voluntária por parte das próprias empresas e promover medidas de autorregulação. Um dos principais desafios consiste, ainda, na passagem de um maior equilíbrio numérico de gênero para uma igualdade substantiva nos conselhos de administração, aumentando ao mesmo tempo a representação das mulheres em cargos de poder e influência efetiva nos processos decisórios.

Sara Falcão Casaca ${ }^{1}$ sarafc@iseg.ulisboa.pt 0000-0002-7944-5007

Maria João Guedes ${ }^{1}$ mjguedes@iseg.ulisboa.pt 0000-0002-4342-2620

Susana Ramalho Marques² smarques@iseg.ulisboa.pt 0000-0001-7116-7252

\section{Nuno Paço ${ }^{1}$}

npaco@iseg.ulisboa.pt 0000-0001-9930-460X

1Universidade de Lisboa, Instituto Superior de Economia e Gestão, Lisboa, Portugal

2Universidade de Lisboa, Centro de Investigação em Sociologia Económica e das Organizações (SOCIUS), Lisboa, Portugal

\section{INTRODUÇÃO}

Este artigo busca analisar o impacto do novo quadro normativo implementado em Portugal com o intuito de obter um maior equilíbrio de gênero em órgãos de poder e tomada de decisão nas empresas, especialmente nas que se encontram cotadas na Bolsa de Valores/Euronext Lisbon (o único segmento considerado neste estudo), e também em empresas estatais e empresas públicas locais. As questões de pesquisa subjacentes ao estudo são: em que medida a lei de cotas de gênero tem efetivamente alcançado o objetivo esperado, isto é, um maior equilíbrio de gênero nos conselhos de administração das empresas cotadas em bolsa? Se a referida lei tem sido bemsucedida, quais são os desafios ainda a ser enfrentados?

Inicia-se com uma breve contextualização do debate sobre a sub-representação das mulheres nos conselhos de administração (WoB, do inglês Women on Boards) e sobre o novo marco de políticas com efeito vinculativo (a chamada lei da cota de gênero), introduzido em alguns países da União Europeia (UE) e em Portugal. Em seguida, são examinados os dados relativos à representação de homens e mulheres nos conselhos de administração das empresas cotadas em bolsa. Conclui-se que a introdução desta medida legislativa de natureza vinculativa em Portugal acelerou o movimento em direção a uma maior representação das mulheres nos conselhos de administração das empresas 
cotadas em bolsa, representando um novo impulso ao progresso neste domínio, porém lento, gerado pelos anteriores incentivos que visaram estimular a ação voluntária por parte das próprias empresas e promover medidas de autorregulação. Um dos principais desafios consiste, ainda, na passagem de um maior equilíbrio numérico de gênero para uma igualdade nos conselhos de administração, aumentando ao mesmo tempo a representação das mulheres em cargos de poder e influência efetiva nos processos decisórios.

Nossa pesquisa encontra-se alinhada com um número crescente de estudos que analisam o impacto de marcos regulatórios que visam promover um maior equilíbrio de gênero nos órgãos de decisão política e econômica. A pesquisa aqui apresentada faz parte de um Projeto mais abrangente sobre Mulheres nos Órgãos de Gestão das Empresas: Uma Abordagem Integrada. ${ }^{1}$

\section{A SUB-REPRESENTAÇÃO DAS MULHERES NOS CONSELHOS DE ADMINISTRAÇÃO: SÍNTESE DAS EXPLICAÇÕES TEÓRICAS}

Embora exista alguma variedade entre as diferentes sociedades, a segregação sexual vertical é um dos padrões mais persistentes de desigualdade de gênero (Terjesen \& Sealy, 2016), pois as mulheres enfrentam barreiras à progressão na carreira em todo o mundo (International Labour Organization [ILO], 2015). As explicações para a sub-representação das mulheres nos conselhos de administração podem ser encontradas em fatores individuais, organizacionais e sociais/institucionais (lannotta, Gatti, \& Huse, 2016; Menéndez, Fagan, \& Ansón, 2012). As explicações baseadas no indivíduo destacam o fato de que as mulheres enfrentam maiores dificuldades para alcançar os cargos mais altos nas organizações porque lhes faltam o capital humano necessário (habilidades e experiência gerencial), uma rede de contatos (o chamado networking) e a predisposição atitudinal certa (falta de ambição de carreira) (Coleman, 1988; Hakim, 2000).

Os contributos institucionalistas, por sua vez, colocam a ênfase nas estruturas, processos e práticas organizacionais discriminatórias (Acker, 1990; Connell, 2006) e na tendência para uma sub-representação das mulheres, a par com o tokenismo (Kanter, 1977), para acentuar os estereótipos de gênero, o favoritismo dentro dos grupos e os mecanismos de exclusão que reproduzem a homofilia e posteriormente confirmar ainda mais a condição das mulheres como outsiders (Konrad, Kramer, \& Erkut, 2008; Lewis \& Simpson, 2012).
De maneira complementar, os argumentos com o foco na sociedade enfatizam o papel do contexto nacional, incluindo a influência das normas culturais e de ordem de gênero. Com base em contribuições oriundas da teoria institucional e da teoria de stakeholders, as análises têm se focado na estrutura normativa e regulatória e no papel que as instituições nacionais e os principais atores sociais desempenham no avanço da igualdade de gênero (Grosvold \& Brammer, 2011; Seierstad, Gabaldon, \& MensiKlarbach, 2017; Terjesen, Aguilera, \& Lorenz, 2015). Assim, um número crescente de estudos, inclusive o nosso, tem examinado os diferentes marcos regulatórios voltados a um maior equilíbrio de gênero nos órgãos de decisão política e econômica, bem como seus respectivos impactos e resultados.

\section{A BASE PRINCIPAL DA AÇÃO AFIRMATIVA NA UE E EM PORTUGAL: REGULAÇÃO DA REPRESENTAÇÃO DE GÊNERO NAS EMPRESAS COTADAS EM BOLSA}

A Convenção das Nações Unidas para a Eliminação de Todas as Formas de Discriminação contra as Mulheres², tida como o documento magno sobre os direitos das mulheres e aprovada na Assembleia Geral da ONU em 1979, exortou todos os Estados-membros a garantirem igualdade substantiva entre homens e mulheres, garantindo participação igual nos processos decisórios em todas as áreas da vida. Em conformidade com a Declaração e Plataforma de Ação de Pequim, assinada na Quarta Conferência Mundial das Nações Unidas sobre a Mulher, em 1995 , a sub-representação feminina nos processos decisórios tem sido integrada em políticas da UE desde meados da década de 1990, as quais têm sido incorporadas em documentos estratégicos importantes (Humbert, Kelan, \& Clayton-Hathway, 2019).

Em linha com a visão de que a ação afirmativa pode contribuir para garantir a igualdade de gênero substantiva e efetiva ao proporcionar às mulheres acesso aos processos decisórios e reforçar a justiça social e a democracia (Lépinard \& Rubio-Marín, 2018), em 2003 , o Conselho Europeu recomendou que todos os governos dos Estadosmembros considerassem o "estabelecimento de metas temporais com vista a alcançar uma participação equilibrada de mulheres e homens na tomada de decisão política e pública" (Council of Europe, trad. 2003). A implementação de alterações legislativas nacionais, incluindo medidas de ação afirmativa, é apoiada pelas políticas centrais da UE. $\mathrm{O}$ artigo 23 da Carta dos Direitos Fundamentais da UE. afirma que "O princípio da igualdade não obsta a que se mantenham

10 projeto é apoiado pela Fundação para a Ciência e Tecnologia (PTDC/SOC-ASO/29895/2017). Site: https://womenonboards.pt

2 Ratificada por Portugal em 1980. 
ou adoptem medidas que prevejam regalias específicas a favor do sexo sub-representado." (European Union, trad. 2000). 0 princípio da ação afirmativa está também incorporado no artigo 157, n. 4, do Tratado sobre o Funcionamento da União Europeia - “A fim de assegurar, na prática, a plena igualdade entre homens e mulheres na vida profissional, o princípio da igualdade de tratamento não obsta a que os Estados-membros mantenham ou adoptem medidas que prevejam regalias específicas destinadas a facilitar o exercício de uma actividade profissional pelas pessoas do sexo sub-representado, ou a prevenir ou compensar desvantagens na sua carreira profissional" (European Union, trad. 2012).

Essa estrutura funcionou como base jurídica para a proposta de 2012 de uma diretiva da UE sobre a melhoria do equilíbrio de gênero entre administradores não executivos de empresas cotadas em bolsa e medidas relacionadas. Um dos principais argumentos para a necessidade de uma diretiva em nível europeu foi a falta de transparência dos procedimentos de seleção e dos critérios de qualificação para cargos nos conselhos de administração. Diferentemente do foco na ligação entre a representação equilibrada de homens e mulheres e uma situação de democracia plena narrativa predominante na UE na década de 1990 -, a proposta de 2012 baseou-se principalmente em uma abordagem de orientação instrumental ao afirmar que "a subutilização das habilidades de mulheres altamente qualificadas constitui uma perda de potencial de crescimento econômico (Elomaki, 2017). A plena mobilização de todos os recursos humanos disponíveis será um elemento fundamental para enfrentar os desafios demográficos da UE, competir com êxito numa economia globalizada e garantir uma vantagem comparativa em relação a países exteriores ao bloco. Além disso, o desequilíbrio de gênero nos conselhos de administração das empresas cotadas em bolsa da UE pode ser uma oportunidade perdida em nível de empresa em termos de governança corporativa e desempenho econômico das empresas" (European Commission, trad., 2012).

Cabe notar que esta proposta ainda está pendente devido a resistências internas expressas por alguns Estados-membros (European Parliament, 2021). Seguindo o efeito bola de neve gerado pela legislação norueguesa de cotas, criada em 2003 e voltada à promoção do equilíbrio de gênero nos conselhos (Machold, Huse, Hansen, \& Brogi, 2013), muitos países europeus e da UE aprovaram legislação sobre cotas de gênero nos conselhos de administração (Humbert et al., 2019; Senden, Kruisinga, Burri, \& Timmer, 2018).

\section{Portugal: Contextualização}

Seguindo a abordagem comparativa institucionalista proposta por Terjesen et al. (2015), foi sugerido anteriormente que Portugal dispõe de condições institucionais favoráveis para a adoção de uma cota de gênero para os conselhos de administração no setor empresarial, incluindo: 1) uma taxa de emprego feminino relativamente elevada e, apesar das fragilidades financeiras inerentes a uma economia semiperiférica, um estado de bemestar cujas políticas estão baseadas no princípio da igualdade de gênero; 2) uma coalizão de governo tendente à esquerda na época em que o projeto de lei foi formulado e proposto no parlamento; 3) e um legado de iniciativas de igualdade de gênero dependentes de caminho na área de políticas públicas (Casaca, 2017).

Portugal tem apresentado uma elevada taxa de emprego feminino (na faixa etária entre 15 e 64 anos) na UE (67,6\%, em 2019, acima da média da UE28 de 64,1\%) (Eurostat, 2020). Este é um padrão há muito estabelecido no país, e é descrito como uma de suas singularidades (Casaca, 2012; Ferreira, 1999; Torres, Silva, Monteiro, \& Cabrita, 2005), uma vez que o comportamento do emprego feminino tem-se aproximado mais daquele dos países nórdicos do que dos países do sul da Europa, onde as taxas de emprego estão entre as mais baixas da UE (Casaca, 2012). Soma-se a isto o maior investimento em educação realizado pelas mulheres em relação aos homens $(61,6 \%$ da população ativa com diploma de ensino superior são mulheres) (Pordata, 2019). A segregação vertical com base no gênero, no entanto, é um dos principais desafios a ser enfrentados em termos do avanço da igualdade de gênero: as mulheres representam $27 \%$ dos indivíduos que atuam como chefes de departamento, enquanto apenas 10\% ocupam cargos de diretoria (Informa D\&B, 2019). De acordo com o Índice de Igualdade de Gênero (European Institute for Gender Equality [EIGE], 2020a), a pontuação de Portugal ainda é fraca no que diz respeito à distribuição de poder no âmbito econômico (medida pela proporção de mulheres nos conselhos de administração das maiores empresas cotadas nas bolsas de valores nacionais e nos conselhos de administração dos Bancos Centrais nacionais), uma vez que a pontuação, em 2018, ainda era de apenas $44,9 \%$. No entanto, a taxa de melhora tem sido notável, pois, em 2015, por exemplo, a proporção de mulheres atuando em conselhos era de apenas $16,4 \%$. A seção seguinte traz a explicação para esse progresso acelerado.

\section{Do lento progresso voluntário ao acelerador regulatório}

A fim de responder à questão-chave da pesquisa - em que medida a lei de cotas de gênero tem sido eficaz em proporcionar o esperado maior equilíbrio de gênero nos conselhos de administração? -, foi recolhida informação publicada na base 
de dados Gender Statistics Database (EIGE, 2020b), a qual disponibiliza estatísticas sobre a representação de homens e mulheres nos conselhos de administração das maiores empresas cotadas nas bolsas de valores da UE. Quando a lei das cotas entrou em vigor, em 2018, 18 empresas estavam cotadas no índice PSI-20, e, atualmente (em outubro de 2020), são 17 as empresas que fazem parte deste mesmo universo.

Estes dados permitem uma comparação entre os avanços em relação à presença de mulheres nos órgãos de gestão das empresas em Portugal e os avanços nos demais países da UE, embora apenas para o segmento específico das maiores empresas cotadas em bolsa. Para todo o universo das empresas deste segmento do país (40 e 38 no total, em 2018 e 2020 , respectivamente), foram coletados dados dos relatórios financeiros e de governança corporativa anuais publicamente disponíveis.

Antes da introdução do novo marco regulatório no país, as políticas de promoção do equilíbrio de gênero nos conselhos de administração contavam com uma combinação de iniciativas de conscientização e medidas brandas, como recomendações de políticas e incentivos à autorregulação, as quais obtiveram impacto limitado sobre a representação numérica das mulheres (Casaca, 2017). Em 2012, quando as mulheres representavam apenas $7,4 \%$ dos membros dos conselhos das maiores empresas cotadas em bolsa, o governo, então liderado por uma coligação de centro-direita (2011-2015), aprovou uma resolução recomendando explicitamente que as empresas cotadas em bolsa adotassem medidas de autorregulação para atingir uma presença plural de mulheres e homens em cargos de gestão e supervisão (nenhuma meta específica foi fixada) (Figura 1, A).

As mudanças mostraram-se muito lentas e, em março de 2015, o mesmo governo convidou todas as empresas cotadas em bolsa a assinarem um termo que expressava seu compromisso em alcançar uma representação mínima de 30\% para cada sexo (Figura 1, B). Em junho de 2015, 13 dentre 43 empresas cotadas em bolsa assinaram o referido termo, com a proporção de mulheres nos conselhos de administração chegando a quase 14\% (Figura 1, C). Um novo governo, apoiado por partidos tendentes à esquerda, que está no poder desde novembro de 2015, anunciou planos de introduzir uma legislação contendo cotas obrigatórias - promessa que cumpriu dois anos depois.

As metas estabelecidas pela lei das cotas de gênero (Lei n. 62/2017) publicada em agosto de 2017 (Figura 1, D) estão bem abaixo do limite mínimo de paridade de $40 \%$ (Council of Europe, 2003), que é também o objetivo quantitativo estabelecido na proposta da Diretiva da UE, apresentada em 2012 (European Commission, 2012). A aplicação desta lei divide-se em duas fases: na primeira fase, a proporção de membros de cada sexo indicados para cada órgão de administração e supervisão não pode ser inferior a $20 \%$ após a primeira assembleia geral eletiva realizada após 1ํ de janeiro de 2018 (Figura 1, E); na segunda fase, tal proporção não poderá ser inferior a 33,3\% após a primeira assembleia geral eletiva realizada após 1ํ de janeiro de 2020 (Figura 1, F). A lei prevê a aplicação de sanções financeiras e de reputação em caso de incumprimento.

Figura 1. Representação das mulheres como membros dos conselhos de administração das maiores empresas cotadas em bolsa (2011-2020)

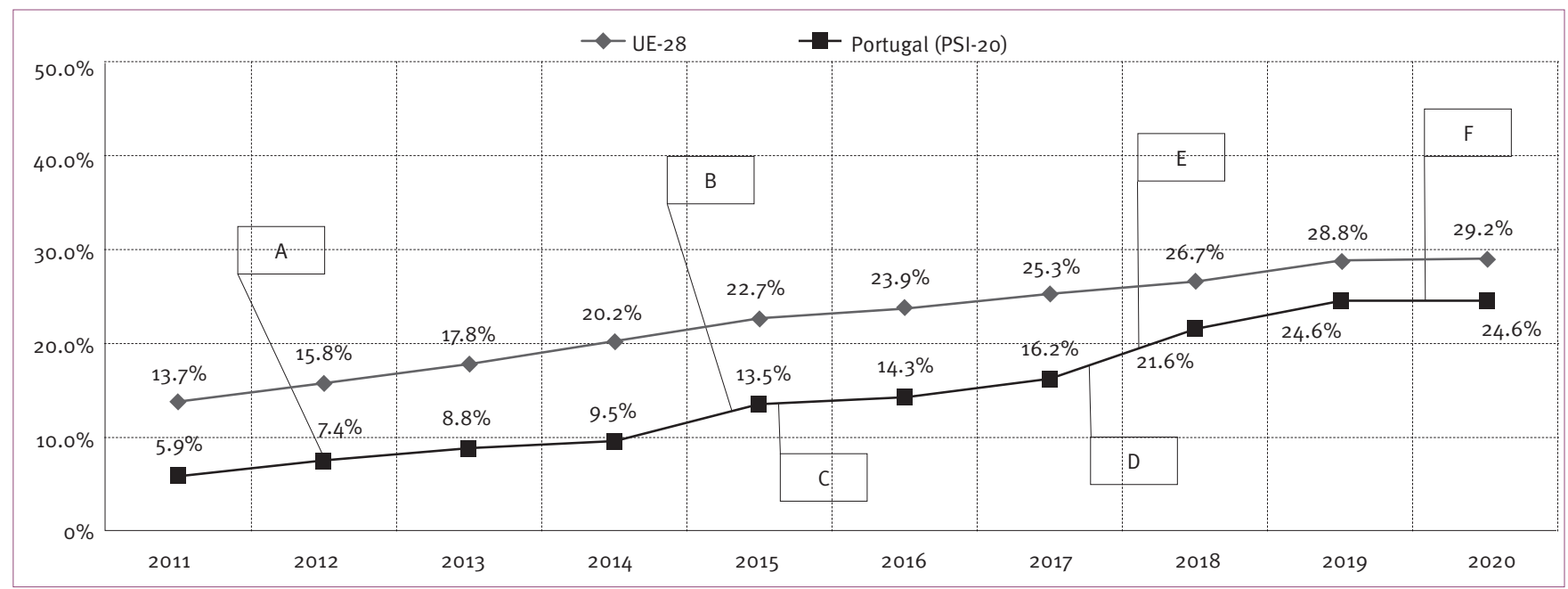

Fonte: EIGE (2020b), Maiores empresas cotadas em bolsa: membros dos conselhos de administração, disponível em: https://eige.europa.eu/gender-statistics/dgs/ indicator/wmidm_bus_bus_wmid_comp_compbm/line [consultado em 25/06/2020] 
A Figura 1 mostra que, após a evolução bastante lenta e inconsistente da passada década, a ambição quantitativa da lei portuguesa está gradualmente produzindo os resultados esperados na composição dos conselhos de administração das empresas-alvo, tendo gerado um aumento da representação feminina nos órgãos de gestão das empresas. Em 2020, a diferença em relação à média da UE-28 é a mais baixa da última década (2011-2020) (cerca de 4 pontos percentuais abaixo). Analisando os avanços alcançados desde 2011 (ano anterior à introdução pelo governo dos incentivos explícitos à adoção de medidas de autorregulação), verifica-se que o aumento mais notável ocorreu em 2018, primeiro ano de aplicação da lei.

Considerando todo o grupo de empresas cotadas em bolsa, quando a lei entrou em vigor, em 2018, a grande maioria dos membros efetivos eram homens ( $82 \%$ ), sendo as mulheres menos de $20 \%$ (18\%) - valor abaixo da proporção verificada no grupo de empresas cotadas no Índice PSI-20. Com relação ao número de empresas já sujeitas a estes requisitos legais, em
2018, após a realização de suas assembleias gerais eletivas na vigência do novo enquadramento legal $(\mathrm{N}=15)$, as mulheres representavam $21 \%$ do total de membros dos conselhos de administração. Em outubro de 2020 , nem todas as empresas cotadas em bolsa desse grupo estavam cumprindo os requisitos legais: em três das 32 empresas, o percentual de mulheres nos órgãos de gestão está abaixo das metas exigidas por lei. A percentagem de mulheres nos conselhos das empresas às quais a lei se aplica aumentou para $28 \%$ em 2020 (Tabela 1). Em termos da natureza do cargo, no entanto, apenas $14 \%$ das mulheres são membros executivos. Quando se trata de cargos não executivos no mesmo grupo de empresas, o nível de representação feminina é maior, somando 37\% do total de membros que ocupam tais cargos, nas empresas cotadas em bolsa e no Índice PSI-20. Além disso, para o mesmo ano e grupo de empresas, apenas uma mulher ocupava o cargo de CEO, enquanto outras duas exerciam a presidência do conselho de administração.

Tabela 1. Percentagem de WoB nas empresas cotadas em bolsa em Outubro de 2020

\begin{tabular}{|c|c|c|c|}
\hline & \multicolumn{3}{|c|}{ WoB (\%) } \\
\hline & Cargos executivos & $\begin{array}{l}\text { Cargos não } \\
\text { executivos }\end{array}$ & Total \\
\hline Total de empresas cotadas em bolsa $(\mathrm{N}=38)$ & $14 \%$ & $35 \%$ & $26 \%$ \\
\hline Empresas às quais se aplica a lei de cotas $(\mathrm{N}=32)$ & $14 \%$ & $37 \%$ & $28 \%$ \\
\hline Empresas às quais se aplica a meta de $20 \%(\mathrm{~N}=22)$ & $16 \%$ & $34 \%$ & $27 \%$ \\
\hline Empresas às quais se aplica a meta de $33,3 \%(\mathrm{~N}=10)$ & $11 \%$ & $49 \%$ & $31 \%$ \\
\hline
\end{tabular}

\section{CONSIDERAÇÕES FINAIS E OS DESAFIOS À FRENTE}

A introdução de uma medida legislativa de natureza vinculativa em Portugal tem funcionado como um acelerador que leva a uma maior representação das mulheres nos conselhos de administração das empresas cotadas em bolsa, conferindo assim um impulso adicional ao progresso de longa data, porém lento, gerado pelos anteriores incentivos que visaram estimular a ação voluntária por parte das próprias empresas e promover medidas de autorregulação. No entanto, nem todas as empresas às quais a lei se aplica estão realmente cumprindo as metas legais, e as sanções deverão ser impostas nos próximos meses. Um tema fundamental em nossa pesquisa é identificar se um maior equilíbrio numérico de gênero está, de fato, promovendo uma igualdade de gênero mais substantiva nos conselhos de administração. Como foi demonstrado, um desafio evidente é aumentar a representação das mulheres em posições de poder e influência sobre os processos decisórios (papéis executivos), visto que a disparidade de gênero ainda é notavelmente grande. A próxima etapa de nossa pesquisa consistirá em examinar a dinâmica social dos conselhos de administração (processos intraconselho) e os resultados de um maior equilíbrio de gênero nos conselhos. Uma vez que a lei de cotas de gênero também torna obrigatória a adoção de Planos para a Igualdade, o foco nos resultados buscará identificar em que medida uma proporção cada vez maior de WoB está estimulando uma revisão das políticas, práticas e processos corporativos internos, induzindo culturas e modelos de organização de trabalho mais inclusivos em termos de igualdade de gênero. 0 projeto atual busca, ainda, integrar teoria, pesquisas, políticas e prática, tendo institucionalizado um think tank no qual importantes stakeholders dedicam-se a 
reflexões baseadas em evidências sobre o equilíbrio de gênero nos conselhos (como os achados do presente estudo) e seu impacto na afirmação dos valores de igualdade de gênero na sociedade. Um desafio fundamental a ser enfrentado consiste na passagem de um maior equilíbrio numérico de gênero para uma igualdade substantiva de gênero nos conselhos de administração, aumentando, ao mesmo tempo, a representação das mulheres em posições de poder e influência sobre os processos decisórios.

\section{REFERÊNCIAS}

Acker, J. (1990). Hierarchies, jobs, bodies: A theory of gendered organizations. Gender and Society, 4(2), 139-158. doi: 10.1177/089124390004002002

Casaca, S. F. (2012). Mercado do trabalho, flexibilidade e relações de género: tendências recentes. In S. F. Casaca (Coord.), Mudanças laborais e relações de género, novos vetores de desigualdade (pp. 9-50). Lisboa, Portugal: Fundação Económicas e Editora Almedina.

Casaca, S. F. (2017). Women on boards in Portugal: The slow progress of the normative framework. In C. Seierstad, P. Gabaldon, \& H. Mensi-Klarbach (Eds.), Gender diversity in the boardroom: European perspectives on increasing female representation (pp. 45-74). London, UK: Palgrave Macmillan.

Coleman, J. S. (1988). Social capital and the creation of human capital. American Journal of Sociology, 94, 95-120.

Connell, R. (2006). Glass ceilings or gendered institutions? Mapping the gender regimes of public sector worksites. Public Administration Review, 66, 792-960. doi: 10.1111/j.1540-6210.2006.00652

Council of Europe. (2003). Balanced participation of women and men in political and public decision-making. Recommendation Rec (2003) 3 of the Committee of Ministers and explanatory memorandum. Retrieved from https://search.coe.int/cm/Pages/result_details. aspx?ObjectID=09000016805e0848

Elomaki, A. (2017). Gender quotas for corporate boards: depoliticizing gender and the economy. Nora - Nordic Journal of Feminist and Research Agenda, 26(1), 53-68.

European Commission. (2012). Proposal for a Directive of the European Parliament and of the Council on improving the gender balance among non-executive directors of companies listed on Stock exchanges and related measures. Retrieved from https://op.europa. eu/en/publication-detail/-/publication/b562b71f-4eob-4boe-a5824609ce5fe428/language-en

European Institute for Gender Equality. (2020a). The Gender Equality Index 2020. Digitalisation and the future of work. Retrieved from https://eige.europa.eu/gender-equality-index/2020

European Institute for Gender Equality. (2020b). Gender statistics database. Retrieved from https://eige.europa.eu/gender-statistics/ dgs/indicator/wmidm_pol_parl__wmid_natparl
European Parliament. (2016). Legislative train 6.2016 gender balance on boards. Retrieved from file://C:/Users/Administrator/Downloads/ area-of-justice-and-fundamental-rights_gender-balance-onboards_2016-06-01.pdf

European Union. (2000). Charter of fundamental rights of the European Union (2000/c 364/01). Retrieved from https://www.europarl. europa.eu/charter/pdf/text_en.pdf

European Union. (2012). Treaty on the functioning of the European Union. Retrieved from https://eur-lex.europa.eu/legal-content/EN/ TXT/PDF/?uri=CELEX:12012E/TXT\&from=EN

Eurostat. (2020). Labour force survey. Database. Retrieved from https:// ec.europa.eu/eurostat/web/lfs/data/database

Ferreira, V. (1999). Os paradoxos da situação das mulheres em Portugal. Revista Crítica de Ciências Sociais, 52/53, 199-227.

Grosvold, J., \& Brammer, S. (2011). National institutional systems as antecedents of female board representation: An empirical study. Corporate Governance: An International Review, 19(2), 116-135. doi: 10.1111/j.1467-8683.2010.00830

Hakim, C. (2000). Work-Lifestyle choices in the 21st century: Preference theory. New York, U.S.: Oxford University Press.

Humbert, A. L., Kelan, E., \& Clayton-Hathway, K. (2019). A rights-based approach to board quotas and how hard sanctions work for gender equality. European Journal of Women's Studies, 26(4), 1-22. doi: $10.1177 / 1350506819857125$

Iannotta, M., Gatti, M., \& Huse, M. (2016). Institutional complementarities and gender diversity on boards: A configurational approach. Corporate Governance: An International Review, 24(4), 406-427. doi: $10.1111 /$ corg.12140

Informa D\&B. (2019). Participação feminina nas empresas em Portugal 2018. Retrieved from https://biblioteca.informadb.pt/files/files/Estudos/retrato-presenca-feminina-2018.pdf

International Labour Organization. (2015). Women in business and management: Gaining momentum. International Labour Office Geneva.

Kanter, R. (1977). Some effects of proportions on group life: Skewed sex ratios and responses to token women. The American Journal of Sociology, 82 (5), 965-990.

Konrad, A. M., Kramer, V., \& Erkut, S. (2008). Critical mass: The impact of three women on corporate boards. Organizational Dynamics, 37(2), 145-164, doi: 10.1016/j.orgdyn.2008.02.005

Lépinard, E., \& Rubio-Marín, R. (2018). The French parity reform. In E. Lépinard, \& R. Rubio-Marín (Eds.), Transforming gender citizenship: The irresistible rise of gender quotas in Europe (pp. 62-93). Cambridge,U.K.: Cambridge University Press.

Lewis, P., \& Simpson, R. (2012). Kanter revisited: Gender, power and (in)visibility. International Journal of Management, 14, 141-158. doi: 10.1111/j.1468-2370.2011.00327.x

Machold, S., Huse, M, Hansen, K., \& Brogi, M. (Eds.). (2013). Getting women on to corporate boards: A snowball staring in Norway. Cheltenham, U.K.: Edward Elgar Publishing. 
Menéndez, M. C. G., Fagan, C., \& Anson, S. G. G. (2012). Introduction. In C. Fagan, M. C. G. Menéndez, \& S. G. Ansón (Eds.), Women on corporate boards and in top management: European trends and policy (pp. 1-17). Basingstoke, U.K.: Palgrave.

Pordata. (2019). População activa por nível de escolaridade completo (INE, Inquérito ao Emprego). Retrieved from https://www.pordata.pt/ Portugal/Popula\%c3\%a7\%c3\%a30+activa+total+e+por+n\%c3\%advel+de+escolaridade+completo-1008

Seierstad, C., Gabaldon, P., \& Mensi-Klarbach, H. (Eds.). (2017). Gender diversity in the boardroom: European perspectives on increasing female representation. London, UK: Palgrave Macmillan.
Senden, L., Kruisinga, S., Burri, S., \& Timmer, A. (2018). Gender-balanced company boards in Europe: A comparative analysis of the regulatory, policy and enforcement approaches in the EU and EEA Member States. Brussels, Belgium: European Commission.

Terjesen, S., Aguilera, R. V., \& Lorenz, R. (2015). Legislating a woman's seat on the board: Institutional factors driving gender quotas for board of directors. Journal of Business Ethics, 128, 233-251.

Terjesen, S., \& Sealy, R. (2016). Board gender quotas: Exploring ethical tensions from a multi-theoretical perspective. Business Ethics Quarterly, 26(1), 23-65. doi: 10.1017/beq.2016.7

Torres, A. C., Silva, F. V., Monteiro, T. L., \& Cabrita, M. (2005), Homens e mulheres entre família e trabalho. Lisboa, Portugal: CITE.

\section{CONTRIBUIÇÕES DOS/AS AUTORES/AS}

Os/as autores/as declaram que participaram de todos os estágios do desenvolvimento do manuscrito. Sara Falcão Casaca trabalhou na conceitualização e na abordagem teórico-metodológica. A revisão teórica foi realizada por Sara Falcão Casaca. A coleta de dados foi coordenada por Sara Falcão Casaca e Maria João Guedes. A análise dos dados teve participação de Susana Ramalho Marques e Nuno Paço. Todos/as os/as autores/as trabalharam juntos/as na escrita e revisão final do manuscrito. 